\title{
Relativistic corrections for the ground electronic state of molecular hydrogen
}

\author{
Mariusz Puchalski and Jacek Komasa \\ Faculty of Chemistry, Adam Mickiewicz University, Umultowska 89b, 61-614 Poznań, Poland
}

Krzysztof Pachucki

Faculty of Physics, University of Warsaw, Pasteura 5, 02-093 Warsaw, Poland

(Dated: September 14, 2018)

\begin{abstract}
We recalculate the leading relativistic corrections for the ground electronic state of the hydrogen molecule using variational method with explicitly correlated functions which satisfy the interelectronic cusp condition. The new computational approach allowed for the control of the numerical precision which reached about 8 significant digits. More importantly, the updated theoretical energies became discrepant with the known experimental values and we conclude that the yet unknown relativistic recoil corrections might be larger than previously anticipated.
\end{abstract}

PACS numbers: 31.30.J-, 12.20.Ds, 31.15.-p

\section{INTRODUCTION}

Theoretical studies of hydrogen molecule is the cornerstone of the molecular quantum mechanics. Due to its simplicity, the achieved precision is the highest among all molecules and still has a potential of significant enhancement. This high precision of theoretical predictions for $\mathrm{H}_{2}$ leads to improved tests of quantum electrodynamics and improved bounds on hypothetical interactions [1]. Moreover, at the $10^{-7} \mathrm{~cm}^{-1}$ precision level the dissociation energy is sensitive to the proton charge radius, which may help to resolve the so called proton radius conundrum [2]. This requires high accuracy calculations of not only nonrelativistic energies, but also leading relativistic $O\left(\alpha^{2}\right)$, QED $O\left(\alpha^{3}\right)$, as well as the higher order corrections $O\left(\alpha^{4}\right)$ and $O\left(\alpha^{5}\right)$. In fact, the nonrelativistic energies can already be calculated with the precision of $10^{-7} \mathrm{~cm}^{-1}$, as demonstrated in Ref. [3]. The $O\left(\alpha^{4}\right)$ contribution has very recently been calculated [4] using explicitly correlated Gaussian (ECG) functions with $1+r_{12} / 2$ prefactor $(r E C G)$ that makes the interelectronic cusp condition to be exactly satisfied. Here, we report the results for the leading $O\left(\alpha^{2}\right)$ relativistic correction using $r$ ECG functions and conclude that the compilation of previous results in Ref. [5] has underestimated numerical uncertainties. We improve the numerical precision by 3-4 orders of magnitude and present in detail our computational approach.

\section{COMPUTATIONAL METHOD}

In the Born-Oppenheimer (BO) approximation the total wave function is assumed to be a product of the electronic and nuclear functions. The Schrödinger equation for the electronic wave functions in the infinite nuclear mass limit (assuming atomic units) is

$$
H \psi\left(\vec{r}_{1}, \vec{r}_{2}\right)=\mathcal{E}(R) \psi\left(\vec{r}_{1}, \vec{r}_{2}\right)
$$

where

$$
H=\frac{1}{2}\left(\vec{p}_{1}^{2}+\vec{p}_{2}^{2}\right)+V
$$

and

$$
V=\frac{1}{R}-\frac{1}{r_{1 A}}-\frac{1}{r_{2 A}}-\frac{1}{r_{1 B}}-\frac{1}{r_{2 B}}+\frac{1}{r}
$$

with $R=r_{A B}$ and $r=r_{12}$, and where indices 1 and 2 correspond to electrons, whereas $A$ and $B$ correspond to the nuclei. The leading relativistic correction in the $\mathrm{BO}$ approximation, is that $\mathcal{E}_{\text {rel }}(R)$ to the nonrelativistic potential $\mathcal{E}(R)$. This correction can be expressed in terms of the expectation value

$$
\mathcal{E}_{\text {rel }}(R)=\left\langle\psi\left|H_{\text {rel }}\right| \psi\right\rangle
$$

of the Breit-Pauli Hamiltonian [6]

$$
\begin{aligned}
H_{\mathrm{rel}}= & -\frac{1}{8}\left(p_{1}^{4}+p_{2}^{4}\right)+\pi \delta^{3}(r)-\frac{1}{2} p_{1}^{i}\left(\frac{\delta^{i j}}{r}+\frac{r^{i} r^{j}}{r^{3}}\right) p_{2}^{j} \\
& +\frac{\pi}{2}\left(\delta^{3}\left(r_{1 A}\right)+\delta^{3}\left(r_{2 A}\right)+\delta^{3}\left(r_{1 B}\right)+\delta^{3}\left(r_{2 B}\right)\right),(5
\end{aligned}
$$

where we neglected spin dependent terms vanishing for the ground electronic state of ${ }^{1} \Sigma_{g}^{+}$symmetry. The accurate calculation of the above expectation value is the principal goal of this work. We assume that every $r$ ECG basis function contains the $1+r / 2$ factor and perform special transformation (regularization) of matrix elements, including those with the Dirac- $\delta$ function [7], for improving the numerical convergence. We demonstrate a significant enhancement in numerical precision and indicate that previous numerical results [5] were not as accurate as claimed. In order to be more convincing, we provide results obtained in three approaches: i) direct (no regularization) with ECG, ii) standard regularization with ECG, and iii) modified regularization with $r$ ECG functions. To test the convergence of these three different approaches, at first we perform calculations for $R=0$, namely for the helium atom, for which highly accurate reference results can be obtained using explicitly correlated exponential functions. Next, the ECG calculations are performed for molecular hydrogen. Comparison of individual operators from different approaches is presented for the equilibrium internuclear distance, namely for $R=1.4$ a.u. The most accurate predictions were obtained for the regularization with $r$ ECG functions at 53 points in the range $R=0.0-10$ a.u. 
Except for our recent paper [4], there has been no similar study of regularization techniques in literature due to difficulties with two-center integrals involving inverse powers of interparticle distances. In relation to this, we have introduced a novel algorithm for numerical quadrature of non-standard ECG two-center integrals [4], which enables very efficient calculations of all complicated matrix elements.

\section{REGULARIZATION OF THE RELATIVISTIC CORRECTION}

In this section we provide regularization formulas for matrix elements with Dirac- $\delta$ and $p^{4}$ operators in $H_{\text {rel }}$. The latter operator can be regularized according to the two schemes: the standard one, already employed in the past in quantum molecular computations, [8] and the modified scheme, valid in the case of the wave function obeying the Kato's cusp condition.

According to the standard scheme the relativistic operators are transformed into the regular form by the following relations

$$
\begin{aligned}
4 \pi \delta^{3}\left(r_{1 A}\right) & =4 \pi\left[\delta^{3}\left(r_{1 A}\right)\right]_{r}+\left\{\frac{2}{r_{1 A}}, H-\mathcal{E}\right\} \\
4 \pi \delta^{3}(r) & =4 \pi\left[\delta^{3}(r)\right]_{r}+\left\{\frac{1}{r}, H-\mathcal{E}\right\} \\
p_{1}^{4}+p_{2}^{4} & =\left[p_{1}^{4}+p_{2}^{4}\right]_{r}+4\{\mathcal{E}-V, H-\mathcal{E}\}+4(H-\mathcal{E})^{2}
\end{aligned}
$$

where

$$
\begin{aligned}
4 \pi\left[\delta^{3}\left(r_{1 A}\right)\right]_{r} & =\frac{4}{r_{1 A}}(\mathcal{E}-V)-\vec{p}_{1} \frac{2}{r_{1 A}} \vec{p}_{1}-\vec{p}_{2} \frac{2}{r_{1 A}} \vec{p}_{2} \\
4 \pi\left[\delta^{3}(r)\right]_{r} & =\frac{2}{r}(\mathcal{E}-V)-\vec{p}_{1} \frac{1}{r} \vec{p}_{1}-\vec{p}_{2} \frac{1}{r} \vec{p}_{2} \\
{\left[p_{1}^{4}+p_{2}^{4}\right]_{r} } & =4(\mathcal{E}-V)^{2}-2 p_{1}^{2} p_{2}^{2} .
\end{aligned}
$$

For the exact wave function $\psi$, which fulfills the electronic Schrödinger equation $(\mathcal{E}-H) \psi=0$, the expectation value identity holds $\langle\psi|\ldots| \psi\rangle=\left\langle\psi\left|[\ldots]_{r}\right| \psi\right\rangle$, since for an arbitrary operator $Q,\langle\psi|\{Q, H-\mathcal{E}\}| \psi\rangle=0$. For an approximate function $\tilde{\psi}$, such expectation values do not vanish, but converge to zero in the limit $\tilde{\psi} \rightarrow \psi$. In practice, the numerical convergence of the regularized form is much faster, so the leading relativistic correction shall be evaluated as

$$
\mathcal{E}_{\text {rel }}(R)=\left\langle\psi\left|\left[H_{\text {rel }}\right]_{r}\right| \psi\right\rangle
$$

with

$$
\begin{aligned}
{\left[H_{\mathrm{rel}}\right]_{r}=} & -\frac{1}{8}\left[p_{1}^{4}+p_{2}^{4}\right]_{r}+\frac{\pi}{2}\left(\left[\delta^{3}\left(r_{1 A}\right)\right]_{r}+\left[\delta^{3}\left(r_{2 A}\right)\right]_{r}\right. \\
& \left.+\left[\delta^{3}\left(r_{1 B}\right)\right]_{r}+\left[\delta^{3}\left(r_{2 B}\right)\right]_{r}\right)+\pi\left[\delta^{3}(r)\right]_{r} \\
& -\frac{1}{2} p_{1}^{i}\left(\frac{\delta^{i j}}{r}+\frac{r^{i} r^{j}}{r^{3}}\right) p_{2}^{j}
\end{aligned}
$$

The modified regularization is applied when the wave function $\tilde{\psi}$ exactly satisfies the interelectronic cusp condition, as for example the $r$ ECG function does. In this case, the action of $\left[p_{1}^{4}+p_{2}^{4}\right]_{r}$ on such a function can be represented as

$$
\left[p_{1}^{4}+p_{2}^{4}\right]_{r}|\tilde{\psi}\rangle=\left\{4(\mathcal{E}-V)^{2}-2 \tilde{p}_{1}^{2} \tilde{p}_{2}^{2}+8 \pi \delta^{3}(r)\right\}|\tilde{\psi}\rangle .
$$

The new term $\tilde{p}_{1}^{2} \tilde{p}_{2}^{2}$ (in contrast to $p_{1}^{2} p_{2}^{2}$ ) is understood as the differentiation $\nabla_{1}^{2} \nabla_{2}^{2}$ of $\tilde{\psi}$ as a function, that is with the omission of the $\delta^{3}(r)$-term. Now, if we are interested in determination of the $\left\langle\tilde{\psi}\left|p_{1}^{4}+p_{2}^{4}\right| \tilde{\psi}\right\rangle$ alone, we can additionally replace the Dirac- $\delta$ operator by its regularized form and obtain the 'fully-regularized' expectation value

$$
\left\langle\psi\left|p_{1}^{4}+p_{2}^{4}\right| \psi\right\rangle=\left\langle\psi\left|4(\mathcal{E}-V)^{2}-2 \tilde{p}_{1}^{2} \tilde{p}_{2}^{2}+8 \pi\left[\delta^{3}(r)\right]_{r}\right| \psi\right\rangle .
$$

We emphasize that here, unlike $p_{1}^{2} p_{2}^{2}$, the $\tilde{p}_{1}^{2} \tilde{p}_{2}^{2}$ term differentiates the right-hand-side wave function only. The specific relationship (14) can also be further employed to simplify the expectation value of the overall Breit-Pauli Hamiltonian (5) by complete elimination of the $\pi \delta^{3}(r)$ term

$$
\begin{aligned}
\mathcal{E}_{\text {rel }}(R)= & \left\langle\psi\left|\left[H_{\text {rel }}\right]_{r}^{\prime}\right| \psi\right\rangle, \\
{\left[H_{\text {rel }}\right]_{r}^{\prime}=} & -\frac{1}{2}(\mathcal{E}-V)\left(\mathcal{E}-\frac{1}{R}-\frac{1}{r}\right) \\
& +\frac{1}{4}\left(\tilde{p}_{1}^{2} \tilde{p}_{2}^{2}+\vec{p}_{1} \tilde{V} \vec{p}_{1}+\overrightarrow{p_{2}} \tilde{V} \vec{p}_{2}\right) \\
& -\frac{1}{2} p_{1}^{i}\left(\frac{\delta^{i j}}{r}+\frac{r^{i} r^{j}}{r^{3}}\right) p_{2}^{j}
\end{aligned}
$$

with $\tilde{V}=-1 / r_{1 A}-1 / r_{1 B}-1 / r_{2 A}-1 / r_{2 B}$. Apart from its compactness, this formula has an additional important advantage which is not readily noticeable. Due to the above cancellations, all the time-consuming integrals with three odd powers of interparticle distances do not appear in the matrix elements with $r E C G$ functions. This non-trivial cancellation has remarkable impact on calculations of the relativistic correction.

\section{INTEGRALS WITH ECG FUNCTIONS}

The variational wave function

$$
\begin{aligned}
\psi & =\sum_{i} c_{i} \psi_{i}\left(\vec{r}_{1}, \vec{r}_{2}\right), \\
\psi_{i} & =(1+\hat{i})\left(1+P_{1 \leftrightarrow 2}\right) \phi_{i}\left(\vec{r}_{1}, \vec{r}_{2}\right),
\end{aligned}
$$

where $\hat{i}$ and $P_{1 \leftrightarrow 2}$ are the inversion and the electron exchange operators, can be accurately represented in the basis of ECG functions of the form

$$
\phi_{\Sigma^{+}}=e^{-a_{1 A} r_{1 A}^{2}-a_{1 B} r_{1 B}^{2}-a_{2 A} r_{2 A}^{2}-a_{2 B} r_{2 B}^{2}-a_{12} r^{2}}
$$

or in the basis of the modified $r$ ECG functions

$$
\phi_{\Sigma^{+}}=\left(1+\frac{r}{2}\right) e^{-a_{1 A} r_{1 A}^{2}-a_{1 B} r_{1 B}^{2}-a_{2 A} r_{2 A}^{2}-a_{2 B} r_{2 B}^{2}-a_{12} r^{2}} .
$$

Nonlinear $a$-parameters are determined variationally for every ECG or $r$ ECG basis function and linear $c$-parameters come 
from the solution of the general eigenvalue problem. The primary advantage of ECG type of functions is that all integrals necessary for the calculations of nonrelativistic and relativistic operators can be evaluated very effectively as described below.

Each matrix element can be expressed as a linear combination of the following ECG integrals

$$
\begin{array}{r}
f\left(n_{1}, n_{2}, n_{3}, n_{4}, n_{5}\right)=\frac{1}{\pi^{3}} \int d^{3} r_{1} \int d^{3} r_{2} r_{1 A}^{n_{1}} r_{1 B}^{n_{2}} r_{2 A}^{n_{3}} r_{2 B}^{n_{4}} r_{12}^{n_{5}} \\
\times e^{-a_{1 A} r_{1 A}^{2}-a_{1 B} r_{1 B}^{2}-a_{2 A} r_{2 A}^{2}-a_{2 B} r_{2 B}^{2}-a_{12} r_{12}^{2}}(22)
\end{array}
$$

with integers $n_{i}$ and real parameters $a$. Among all the integrals represented by the above formula we can distinguish two subsets that can be evaluated analytically. The first subset contains the regular ECG integrals with the non-negative even integers $n_{i}$ such that $\sum_{i} n_{i} \leq \Omega_{1}$, where the shell parameter $\Omega_{1}=0,2,4, \ldots$ These integrals can be generated by differentiation over $a$-parameters of the following master integral

$$
f(0,0,0,0,0)=X^{-3 / 2} e^{-R^{2} \frac{Y}{X}}
$$

where

$$
\begin{aligned}
X= & \left(a_{1 A}+a_{1 B}+a_{12}\right)\left(a_{2 A}+a_{2 B}+a_{12}\right)-a_{12}^{2} \\
Y= & \left(a_{1 B}+a_{1 A}\right) a_{2 A} a_{2 B}+a_{1 A} a_{1 B}\left(a_{2 A}+a_{2 B}\right) \\
& +a_{12}\left(a_{1 A}+a_{2 A}\right)\left(a_{1 B}+a_{2 B}\right) .
\end{aligned}
$$

Each differentiation raises one of the $n_{i}$ exponents by two. The second subset of integrals permits a single odd index $n_{i} \geq-1$ for which $\sum_{i} n_{i} \leq \Omega_{2}\left(\Omega_{2}=-1,1,3, \ldots\right)$. These so called Coulomb ECG integrals can also be obtained analytically by differentiation of another master integral. For instance, when $n_{1}=-1$ the master integral reads

$$
f(-1,0,0,0,0)=\frac{1}{X \sqrt{X_{1}}} e^{-R^{2} \frac{Y}{X}} F\left[R^{2}\left(\frac{Y_{1}}{X_{1}}-\frac{Y}{X}\right)\right],
$$

where $X_{1}=\partial_{a_{1 A}} X, Y_{1}=\partial_{a_{1 A}} Y$, and $F(x)=\operatorname{erf}(x) / x$.

In the standard use of ECG functions the regular integrals with $\Omega_{1}=2$ and Coulomb with $\Omega_{2}=-1$ are sufficient to evaluate matrix elements of the electronic Schrödinger equation (1) and thus to perform calculations of the nonrelativistic energy of the ground state in molecular hydrogen. If additionally the analytic gradient minimization is employed, the integrals with $\Omega_{1}=4$ and $\Omega_{2}=1$ are required. Such nonrelativistic calculations have been widely used for many atomic and molecular systems. [9]

The molecular ECG integrals, as opposed to the atomic ones, have no known analytic form when two or more $n_{i}$-s are odd. Such extended integrals originate from regularization of the relativistic operators, for example from $V^{2}$ in Eq. (11), or from matrix elements of the nonrelativistic Hamiltonian with $r$ ECG basis. The algorithm for numerical evaluation of this extended type of integrals relies on the following relation, which decreases one of the indices by one

$$
\begin{aligned}
& f\left(n_{1}-1, n_{2}, n_{3}, n_{4}, n_{5}\right)= \\
& \left.\frac{2}{\sqrt{\pi}} \int_{0}^{\infty} \mathrm{d} y f\left(n_{1}, n_{2}, n_{3}, n_{4}, n_{5}\right)\right|_{a_{1 A} \rightarrow a_{1 A}+y^{2}} .
\end{aligned}
$$

The right-hand-side $f$ is understood as the integral $f\left(n_{1}, n_{2}, n_{3}, n_{4}, n_{5}\right)$ evaluated with the $a_{1 A}$ parameter replaced by $a_{1 A}+y^{2}$. The transformation $y=-1+1 / x$ converts the infinite integration domain to the finite interval $(0,1)$ for which an $m$-point generalized Gaussian quadrature with logarithmic end-point singularity [10] is applied

$$
\begin{aligned}
& \int_{0}^{1} d x\left[W_{1}(x)+\ln (x) W_{2}(x)\right] \\
& =\sum_{i=1}^{m} w_{i}\left[W_{1}\left(x_{i}\right)+\ln \left(x_{i}\right) W_{2}\left(x_{i}\right)\right] .
\end{aligned}
$$

The $W_{1,2}$ are arbitrary polynomials of maximal degree $m-1$, $w_{i}$ are weights, and $x_{i}$ are nodes. In terms of this quadrature the integral 27) can be approximated by the formula

$$
\begin{aligned}
f\left(n_{1}-1, n_{2},\right. & \left.n_{3}, n_{4}, n_{5}\right)=\frac{2}{\sqrt{\pi}} \sum_{i=1}^{m} w_{i}\left(y_{i}+1\right)^{2} \\
& \times\left. f\left(n_{1}, n_{2}, n_{3}, n_{4}, n_{5}\right)\right|_{a_{1 A} \rightarrow a_{1 A}+y_{i}^{2}} .
\end{aligned}
$$

This quadrature is very efficient for extended integrals with two odd indices, for which typically only $m=30$ nodes allows about 16 significant digits to be obtained. These extended integrals are sufficient not only for all the relativistic operators with the regularization applied to ECG wave function, but also for the modified regularization (16)-17) of $H_{\text {rel }}$ with $r$ ECG wave function.

Nevertheless, calculations with $r$ ECG wave function of the expectation values of the individual relativistic operators involve extended integrals with three odd indices. They can be obtained by the double numerical integration of Coulomb ECG integrals over $30^{2}$ nodes to achieve numerical precision of about 16 significant digits. This two dimensional integration is numerically stable, but time consuming.

\section{CALCULATIONS OF RELATIVISTIC CORRECTIONS}

Relativistic corrections to the $\mathrm{BO}$ potential were calculated according to Eqs. (4), (12), and (16). In order to demonstrate the convergence of these three different approaches with ECG functions we compared results at $R=0$, i.e. for the helium atom, to the results obtained with explicitly correlated exponential (ECE) functions. Calculations with ECE functions are well known in literature (see e.g. Refs. [11] and [12]) and may serve as an excellent reference point and a rigorous test of the convergence of ECG results. Numerical values presented in Table I were obtained with 128, 256, 512, and 1024 ECG basis functions. Direct and standard regularization methods were used with ECG functions whereas the modified regularization with $r$ ECG functions. We observe a significant enhancement of numerical convergence of relativistic operators obtained with $r$ ECG basis. The total relativistic correction with $N=1024$ is accurate to 9 digits in the $r$ ECG basis, and to 5-6 digits in the ECG basis. A similar enhancement is observed for $\mathrm{H}_{2}$ at $R=1.4$ in Table $\amalg$ where we compared our 
results for Dirac- $\delta$ functions with those obtained in the ECE basis. The accuracy of the extrapolated value for the total relativistic correction is estimated to have at least 8 significant digits after the decimal dot. In Table III we provide results for the nonrelativistic energy $\mathcal{E}$, for the relativistic correction $\mathcal{E}_{\text {rel }}$, and also for all the four individual components of the relativistic correction evaluated at $R \in(0,10)$ a.u. with the 1024-term basis of $r$ ECG functions. Our results for the overall relativistic correction $\mathcal{E}_{\text {rel }}$ for $\mathrm{H}_{2}$ are estimated to have 8 significant decimal digits.

\section{VIBRATIONAL AVERAGING}

In order to obtain the final value of the $\alpha^{2}$ relativistic component of the dissociation energy, we solved the radial Schrödinger equation for two potentials. The first potential, used as a reference, is the nonrelativistic $(\mathrm{BO})$ potential $\mathcal{E}(R)$ [14], which yielded the nonrelativistic (BO) energy level $E$. The second was the potential augmented by the relativistic correction $\mathcal{E}_{\text {rel }}(R)$, which gave the eigenvalue corresponding to the relativistic energy level $E+E^{(2)}$. The difference between both the eigenvalues $E^{(2)}$ is the relativistic correction to molecular levels.

To establish reliable uncertainties for the final results we studied two sources of error: the convergence of single point calculations and the polynomial interpolation. As mentioned above, the relativistic correction was evaluated using basis sets of increasing size, which permitted a detailed analysis of the convergence at each internuclear distance (see Tab. II). From this analysis we estimated that in the vicinity of the equilibrium distance the $\mathcal{E}_{\text {rel }}(R)$ bears an uncertainty of $7 \cdot 10^{-9}$ a.u. equivalent to $2 \cdot 10^{-8} \mathrm{~cm}^{-1}$. The influence of the density of the points, at which $\mathcal{E}_{\text {rel }}(R)$ was evaluated, on the accuracy of the final result was assessed by doubling the number of points, which however were calculated only with 512-term basis. As a consequence $E^{(2)}$ was shifted by $5 \cdot 10^{-7} \mathrm{~cm}^{-1}$. The related uncertainty due to the selection of the degree of the interpolation polynomial was also investigated. By changing the degree in the range $5-12$, we observed changes in the relativistic correction at the level of $10^{-7} \mathrm{~cm}^{-1}$. To summarize, the largest contribution to the uncertainty of the relativistic correction $E^{(2)}$ comes from the limited number of points (and the necessity of interpolation) at which the relativistic potential was evaluated. The final relative uncertainty is assumed to be smaller than $10^{-6} \mathrm{~cm}^{-1}$.

\section{RESULTS AND SUMMARY}

Results of our calculations for the dissociation energy and the two selected most accurately measured transitions in $\mathrm{H}_{2}$ and $\mathrm{D}_{2}$ are presented in Tables $[\mathrm{V}$ and $\mathrm{V}$. The nonrelativistic energy $E$ for $\mathrm{H}_{2}$ was calculated by solving the full nonadiabatic Schrödinger equation in the exponential basis [3], whereas for $\mathrm{D}_{2}$ using the NAPT[16] expansion with the neglect of $O(1 / \mu)^{3}$ terms. All the corrections were obtained within the adiabatic approximation. The relativistic correc- tion $E^{(2)}$ was evaluated and reported in this work. The leading QED correction $E^{(3)}$ was obtained in Ref. [17], while the higher order QED, namely $E^{(4)}$ in Ref. [4]. $E^{(5)}$ was estimated from the correction analogous to that of atomic hydrogen with the assumption that it is proportional to the electronnucleus Dirac- $\delta$ and the related uncertainty was assigned to be $50 \%$.

Our results for the leading relativistic corrections significantly differ from those by Piszczatowski et al. [5], whose compilation partially relies on the former calculations by Wolniewicz [8]. For example, our relativistic correction to $D_{0}$ of $\mathrm{H}_{2}$ is $-0.533121(1) \mathrm{cm}^{-1}$ whereas Piszczatowski et al. reported $-0.5319(3) \mathrm{cm}^{-1}$. Interestingly, our result is closer to that by Wolniewicz $-0.5330 \mathrm{~cm}^{-1}$, despite the differences at the level of individual operators. In our opinion, these differences come from the much more accurate calculation of relativistic matrix elements performed here.

Most importantly, our final theoretical predictions for $D_{0}$ are now in disagreement with experimental values, in contrast to the previous theoretical results [5]. This disagreement, most probably, comes from the underestimation of the relativistic nuclear recoil correction. We have previously assumed that these corrections are of the order of the ratio of the electron mass to the reduced mass of the nuclei, which for $\mathrm{H}_{2}$ is $\sim 10^{-3}$. This might be incorrect, because the nonrecoil relativistic correction is anomalously small. This assertion is supported by the example of helium atom. The nonrecoil relativistic correction to the ${ }^{4} \mathrm{He}$ ionization energy is 16904.024 $\mathrm{MHz}$, while the nuclear recoil is $-103.724 \mathrm{MHz}$, so the ratio is $6 \cdot 10^{-3}$ that is an order of magnitude higher than the estimate based on the helium mass ratio $10^{-4}$. On the other hand, in the separated atoms limit the relativistic recoil correction exactly vanishes. Therefore at present this correction cannot be reliably estimated.

If the relativistic nuclear recoil correction in $\mathrm{H}_{2}$ is underestimated, the difference between our predictions and the experimental values for $\mathrm{D}_{2}$ should be smaller than that for $\mathrm{H}_{2}$ and this is really the case (vide Tables IV and VD. We emphasize that our theoretical predictions should be treated as preliminary until the relativistic nuclear recoil corrections are reliably calculated. In fact, such corrections have already been obtained by Stanke and Adamowicz [19] for purely vibrational states. However, their result for the total relativistic dissociation energy of $\mathrm{H}_{2}$ (with $E^{(2)}=-0.5691 \mathrm{~cm}^{-1}$ ), when augmented by missing higher order corrections, yields $D_{0}=36118.0318 \mathrm{~cm}^{-1}$, which differs from the experimental value by as much as $0.038 \mathrm{~cm}^{-1}$, so its numerical uncertainty is out of control. We plan to calculate these nonadiabatic corrections using the fully nonadiabatic wave function in exponential basis as in Ref. [3] or by using nonadiabatic perturbation theory (NAPT) [13]. Certainly, this calculation has to be performed for resolving discrepancies with $\mathrm{H}_{2}$ experiments.

In conclusion, the former excellent agreement of theoretical predictions with experimental $D_{0}$ values was accidental and the improved calculations of the leading relativistic corrections result in a few $\sigma$ disagreements with experimental values for dissociation energies and transition energies, which most 
probably is caused by the unknown relativistic nuclear recoil (nonadiabatic) effects in the relativistic corrections.

\section{ACKNOWLEDGMENTS}

We wish to thank Bogumił Jeziorski for comments to the manuscript. This work was supported by the National Science
Center (Poland) Grant Nos. 2012/04/A/ST2/00105 (K.P.) and 2014/13/B/ST4/04598 (M.P. and J.K.), as well as by a computing grant from the Poznan Supercomputing and Networking Center, and by PL-Grid Infrastructure.
[1] W. Ubachs, J.C.J. Koelemeij, K.S.E. Eikema, E.J. Salumbides, J. Mol. Spectr. 320, 1 (2016).

[2] R. Pohl, A. Antognini, F. Nez, F. D. Amaro, F. Biraben, J. M. R. Cardoso, D. S. Covita, A. Dax, S. Dhawan, L. M. P. Fernandes, et al., Nature 466, 213 (2010).

[3] K. Pachucki and J. Komasa, J. Chem. Phys. 144, 164306 (2016).

[4] M. Puchalski, J. Komasa, P. Czachorowski, and K. Pachucki, Phys. Rev. Lett. 117, 263002 (2016).

[5] K. Piszczatowski, G. Lach, M. Przybytek, J. Komasa, K. Pachucki, and B. Jeziorski, J. Chem. Theory Comput. 5, 3039 (2009).

[6] H. A. Bethe and E. E. Salpeter, Quantum Mechanics of Oneand Two-Electron Systems (Springer-Verlag, Berlin and New York, 1957).

[7] R. J. Drachman, J. Phys. B 14, 2733 (1981).

[8] L. Wolniewicz, J. Chem. Phys. 99, 1851 (1993).

[9] J. Mitroy, S. Bubin, W. Horiuchi, Y. Suzuki, L. Adamowicz, W. Cencek, K. Szalewicz, J. Komasa, D. Blume, and K. Varga, Rev. Mod. Phys. 85, 693 (2013).

[10] K. Pachucki, M. Puchalski, and V. Yerokhin, Computer Physics Communications 185, 2913 (2014).

[11] V. I. Korobov, Phys. Rev. A 66, 024501 (2002).

[12] G. W. F. Drake, M. M. Cassar, and R. A. Nistor, Phys. Rev. A
65, 054501 (2002).

[13] K. Pachucki and J. Komasa, J. Chem. Phys. 130, 164113 (2009).

[14] K. Pachucki, Phys. Rev. A 82, 032509 (2010).

[15] K. Pachucki and J. Komasa, J. Chem. Phys. 141, 224103 (2014).

[16] K. Pachucki and J. Komasa, J. Chem. Phys. 143, 034111 (2015).

[17] J. Komasa, K. Piszczatowski, G. Łach, M. Przybytek, B. Jeziorski, and K. Pachucki, J. Chem. Theory Comput. 7, 3105 (2011).

[18] J. Liu, E. J. Salumbides, U. Hollenstein, J. C. J. Koelemeij, K. S. E. Eikema, W. Ubachs, and F. Merkt, J. Chem. Phys. 130, 174306 (2009).

[19] M. Stanke and L. Adamowicz, J. Phys. Chem. A 117, 10129 (2013).

[20] C.-F. Cheng, Y. R. Sun, H. Pan, J. Wang, A.-W. Liu, A. Campargue, and S.-M. Hu, Phys. Rev. A 85, 024501 (2012).

[21] M. Niu, E. Salumbides, G. Dickenson, K. Eikema, and W. Ubachs, J. Mol. Spectrosc. 300, 44 (2014).

[22] J. Liu, D. Sprecher, C. Jungen, W. Ubachs, and F. Merkt, J. Chem. Phys. 132, 154301 (2010).

[23] D. Mondelain, S. Kassi, T. Sala, D. Romanini, D. Gatti, and A. Campargue, J. Mol. Spectrosc. 326, 5 (2016). 
TABLE I. Convergence of matrix elements of relativistic operators at $R=0$ a.u. (helium atom limit).

\begin{tabular}{|c|c|c|c|}
\hline Basis & Direct & Standard regularization & $r$ ECG (+modified regularization) \\
\hline 128 & & 368357561 & -2.903724366011805 \\
\hline 512 & & 377031170 & -2.903724377030040 \\
\hline 1024 & & 377034103 & -2.903724377034089 \\
\hline \multicolumn{4}{|c|}{$p_{1}^{4}+p_{2}^{4}$} \\
\hline 128 & 108.103812847 & 108.178260879 & 108.175893984 \\
\hline 256 & 108.149717136 & 108.176705311 & 108.176119036 \\
\hline 512 & 108.171069063 & 108.176261126 & 108.176133296 \\
\hline 1024 & 108.174593664 & 108.176173934 & 108.176134411 \\
\hline \multicolumn{4}{|c|}{$\delta^{3}\left(r_{1}\right)+\delta^{3}\left(r_{2}\right)$} \\
\hline 128 & 3.61807292223 & 3.62085592707 & 3.62085250490 \\
\hline 256 & 3.61983231429 & 3.62085832771 & 3.62085826322 \\
\hline 512 & 3.62066249308 & 3.62085862304 & 3.62085861086 \\
\hline 1024 & 3.62079894559 & 3.62085863628 & 3.62085863616 \\
\hline$\infty$-Slater ${ }^{a}$ & & & $3.62085863700(1)$ \\
\hline \multicolumn{4}{|c|}{$\delta^{3}(r)$} \\
\hline 128 & 0.106521423626 & 0.106345075042 & 0.106345517181 \\
\hline 256 & 0.106391759156 & 0.106345347318 & 0.106345416874 \\
\hline 512 & 0.106355477797 & 0.106345369617 & 0.106345375554 \\
\hline 1024 & 0.2 & & 0.27818938036 \\
\hline$\infty$-Slater ${ }^{a}$ & & & $0.27818938108(1)$ \\
\hline \multicolumn{4}{|c|}{$\mathcal{E}_{\text {rel }}$} \\
\hline 128 & -1.95091394168 & -1.95203089354 & -1.95174292889 \\
\hline 256 & -1.95153123551 & -1.95182724821 & -1.95175366041 \\
\hline 512 & -1.95170604993 & -1.95177065311 & -1.95175469640 \\
\hline 1024 & -1.95173983039 & -1.95175970900 & -1.95175476523 \\
\hline$\infty$-Slater ${ }^{a}$ & & & $-1.951754768(1)$ \\
\hline
\end{tabular}

${ }^{a}$ The reference values were evaluated with the atomic ECE basis functions $\phi=\exp \left(-\alpha r_{1}-\beta r_{2}-\gamma r\right)$. 
TABLE II. Convergence of matrix elements of relativistic operators at the equilibrium distance $R=1.4$ a.u.

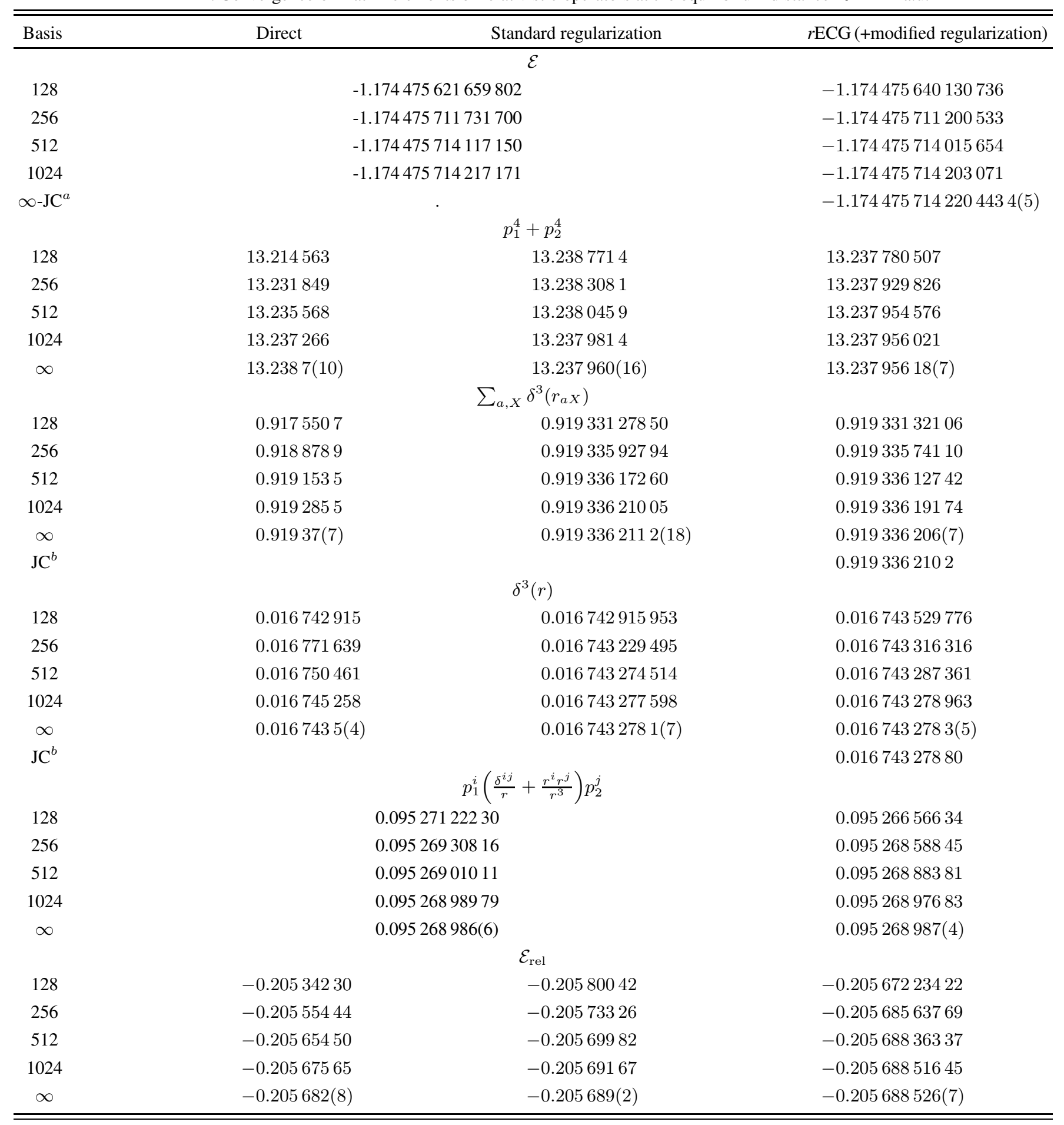

${ }^{a}$ Evaluated with James-Coolidge wave function, Ref. [14].

${ }^{b}$ Evaluated in this work. 
TABLE III. The electronic energy $\mathcal{E}$, expectation values of individual relativistic operators, and the relativistic correction of Eq. (16) evaluated with 1024-term $r$ ECG basis for $\mathrm{H}_{2}$ (all entries in a.u.).

\begin{tabular}{|c|c|c|c|c|c|c|}
\hline$R$ & $\mathcal{E}$ & $p_{1}^{4}+p_{2}^{4}$ & $\sum_{a, X} \delta^{3}\left(r_{a X}\right)$ & $\delta^{3}(r)$ & $p_{1}^{i}\left(\frac{\delta^{i j}}{r}+\frac{r^{i} r^{j}}{r^{3}}\right) p_{2}^{j}$ & $\mathcal{E}_{\text {rel }}$ \\
\hline 0.0 & $\infty$ & 108.17613441 & 7.2417172739 & 0.106345370636 & 0.27818938106 & -1.951754765 \\
\hline 0.05 & 17.1048405957338 & 97.88343711 & 6.4890431775 & 0.105003823908 & 0.27626636142 & -1.850717847 \\
\hline 0.1 & 7.1272167311799 & 87.35944120 & 5.7705971486 & 0.101570564053 & 0.27127375954 & -1.672040901 \\
\hline 0.2 & 2.1978032952426 & 69.10609540 & 4.5689318402 & 0.091368192666 & 0.25582595608 & -1.302271866 \\
\hline 0.4 & -0.1202303411732 & 44.92674848 & 3.0079742620 & 0.068873279242 & 0.21811509421 & -0.783614393 \\
\hline 0.6 & -0.7696354294740 & 31.45554441 & 2.1355542202 & 0.050763240175 & 0.18303648908 & -0.509463149 \\
\hline 0.8 & -1.0200566663407 & 23.55141269 & 1.6165590023 & 0.037667878063 & 0.15386526748 & -0.363237148 \\
\hline 1.0 & -1.1245397195256 & 18.63189259 & 1.2881958110 & 0.028345276112 & 0.13021059568 & -0.281549312 \\
\hline 1.1 & -1.1500573677202 & 16.86266910 & 1.1685384371 & 0.024725634292 & 0.12012130961 & -0.254680537 \\
\hline 1.2 & -1.1649352434217 & 15.41702626 & 1.0699189128 & 0.021643994354 & 0.11101250931 & -0.234013225 \\
\hline 1.3 & -1.1723471490151 & 14.22596331 & 0.9879494323 & 0.019008309934 & 0.10276409419 & -0.218043755 \\
\hline 1.4 & -1.1744757142023 & 13.23795602 & 0.9193361917 & 0.016743278963 & 0.09526897686 & -0.205688516 \\
\hline 1.4011 & -1.1744759313760 & 13.22806227 & 0.9186457757 & 0.016720183220 & 0.09519036690 & -0.205569553 \\
\hline 1.45 & -1.1740570714499 & 12.80752610 & 0.8892304836 & 0.015730076395 & 0.09177395700 & -0.200610272 \\
\hline 1.5 & -1.1728550795518 & 12.41401628 & 0.8615729317 & 0.014787413515 & 0.08843282423 & -0.196156821 \\
\hline 1.6 & -1.1685833733462 & 11.72427668 & 0.8127287476 & 0.013090471717 & 0.08217314696 & -0.188864898 \\
\hline 1.7 & -1.1624587268749 & 11.14559108 & 0.7712986957 & 0.011611319478 & 0.07641801659 & -0.183376700 \\
\hline 1.8 & -1.1550687375868 & 10.65982714 & 0.7360971574 & 0.010316180045 & 0.07110490035 & -0.179362896 \\
\hline 1.9 & -1.1468506970016 & 10.25263362 & 0.7061806011 & 0.009177235715 & 0.06617943868 & -0.176571891 \\
\hline 2.0 & -1.1381329571022 & 9.91253644 & 0.6807907434 & 0.008171495990 & 0.06159453696 & -0.174809213 \\
\hline 2.1 & -1.1291638360667 & 9.63026686 & 0.6593121815 & 0.007279910924 & 0.05730939671 & -0.173922389 \\
\hline 2.2 & -1.1201321168151 & 9.39825583 & 0.6412403665 & 0.006486644310 & 0.05328892414 & -0.173790035 \\
\hline 2.3 & -1.1111817651695 & 9.21024640 & 0.6261570146 & 0.005778509183 & 0.04950304198 & -0.174313461 \\
\hline 2.4 & -1.1024226059759 & 9.06099378 & 0.6137109920 & 0.005144501580 & 0.04592628003 & -0.175410463 \\
\hline 2.5 & -1.0939381299201 & & & 0.004575430210 & 0.04253733371 & -0.177010457 \\
\hline 2.6 & -1.0857912373625 & 8.86147693 & 5750005 & 0.004063603931 & 0.03931882634 & -0.179 \\
\hline 2.7 & -1.0780284841479 & & & 0.003602589090 & 0.03625688742 & -0.1814 \\
\hline 2.8 & -1.0706832334498 & 8.77018399 & 0.5848665704 & 0.003186984950 & 0.03334102116 & -0.184225040 \\
\hline 2.9 & -1.0637780087717 & 8.75745814 & 0.5817929471 & 0.002812250937 & 0.03056370383 & -0.187250948 \\
\hline 3.0 & -1.0573262688383 & 8.76301572 & 0.5800014505 & 0.002474548852 & 0.02792005906 & -0.190498823 \\
\hline 3.2 & -1.0457996613902 & 8.81876665 & 0.5796085282 & 0.001897578330 & 0.02302537071 & -0.197450152 \\
\hline 3.4 & -1.0360753951531 & 8.91801344 & 0.5824483317 & 0.001434600771 & 0.01865530993 & -0.204664704 \\
\hline 3.6 & -1.0280463083390 & 9.04310919 & 0.5873998954 & 0.001068345909 & 0.01482089794 & -0.211757192 \\
\hline 3.8 & -1.0215497953795 & & & 0.000783739899 & 0.01152959176 & -0.218405205 \\
\hline 4.0 & -1.0163902529178 & 9.31327923 & 0.5999421995 & 0.000566901578 & 0.00877312169 & -0.224378488 \\
\hline 4.2 & -1.0123599596533 & 9.43806455 & 0.6061874544 & 0.000404933730 & 0.00652243889 & -0.229550124 \\
\hline 4.4 & -1.0092565162188 & 9.54839599 & 0.6118724265 & 0.000286163107 & 0.00472959559 & -0.233888328 \\
\hline 4.6 & -1.0068952237883 & 9.64234777 & 0.6168193211 & 0.000200467812 & 0.00333424931 & -0.237433284 \\
\hline 4.8 & -1.0051160060122 & 9.72004626 & 0.6209804279 & 0.000139467088 & 0.00227142658 & -0.240269571 \\
\hline 5.0 & -1.0037856585418 & 9.78286262 & 0.6243914654 & 0.000096514570 & 0.00147803156 & -0.242501813 \\
\hline 5.2 & -1.0027968162807 & 9.83276296 & 0.6271329697 & 0.000066524898 & 0.00089718478 & -0.244236803 \\
\hline 5.4 & -1.0020650571894 & 9.87187041 & 0.6293032907 & 0.000045719642 & 0.00048029837 & -0.245573020 \\
\hline 5.6 & -1.0015252518178 & 9.90220197 & 0.6310015807 & 0.000031354765 & 0.00018750516 & -0.246595529 \\
\hline 5.8 & -1.0011278808276 & 9.92554176 & 0.6323187721 & 0.000021470798 & -0.00001291240 & -0.247374807 \\
\hline 6.0 & -1.0008357076028 & 9.94339293 & 0.6333334205 & 0.000014686948 & -0.00014560665 & -0.247967362 \\
\hline 6.5 & -1.0004005479461 & 9.97147881 & 0.6349460878 & 0.000005668125 & -0.00029318606 & -0.248899471 \\
\hline 7.0 & -1.0001979144266 & 9.98548243 & 0.6357607627 & 0.000002183134 & -0.00030790117 & -0.249373815 \\
\hline 7.5 & -1.0001021060380 & 9.99243990 & 0.6361697989 & 0.000000839980 & -0.00027491015 & -0.249621706 \\
\hline 8.0 & -1.0000556046110 & 9.99592278 & 0.6363762591 & 0.000000323028 & -0.00023037686 & -0.249756677 \\
\hline 8.5 & -1.0000321717017 & 9.99770001 & 0.6364822180 & 0.000000124123 & -0.00018810444 & -0.249834155 \\
\hline 9.0 & -1.0000197816909 & 9.99863262 & 0.6365379911 & 0.000000047661 & -0.00015228986 & -0.249881211 \\
\hline 9.5 & -1.0000128559933 & 9.99914248 & 0.6365684974 & 0.000000018282 & -0.00012334587 & -0.249911507 \\
\hline 10.0 & -1.0000087556935 & 9.99943461 & 0.6365860088 & 0.000000007007 & -0.00010039054 & -0.249932046 \\
\hline$\infty$ & -1.0 & 10.0 & 0.6366197723 & 0.0 & 0.0 & -0.25 \\
\hline
\end{tabular}


TABLE IV. Contributions to the dissociation energy $D_{0}$ and two selected most accurate experimental transitions in $\mathrm{H}_{2}\left(\right.$ in $\left.\mathrm{cm}^{-1}\right)$. There are additional $10^{-3}$ relative uncertainties on $E^{(2)}, E^{(3)}$, and $E^{(4)}$ terms due to the BO approximation, which are included in the final result only.

\begin{tabular}{lccc}
\hline \hline Contrib. & $D_{0}$ & $S_{3}(3)$ & $Q_{1}(0)$ \\
\hline$E$ & $36118.79774612(5)$ & $12559.749919(1)$ & $4161.1640703(1)$ \\
$E^{(2)}$ & $-0.533121(1)^{a}$ & 0.065366 & 0.023397 \\
$E^{(3)}$ & $-0.1948(2)$ & $-0.06573(6)$ & $-0.02129(2)$ \\
$E^{(4)}$ & $-0.002067(6)$ & -0.000599 & -0.000192 \\
$E^{(5)}$ & $0.00012(6)$ & $0.000037(19)$ & $0.000012(6)$ \\
$E_{\mathrm{FS}}$ & -0.000031 & -0.000010 & -0.000003 \\
Total & $36118.0678(6)$ & $12559.74898(8)$ & $4161.16599(3)$ \\
Exp. & $36118.06962(37)^{b}$ & $12559.74952(5)^{c}$ & $4161.16636(15)^{d}$ \\
Diff. & 0.0018 & 0.00054 & 0.00037 \\
\hline \hline
\end{tabular}

${ }^{a}$ For comparison, Wolniewicz[8] obtained -0.5330 , Piszczatowski et al.[5] -0.5319 , and Stanke et al.[19] $-0.5691 \mathrm{~cm}^{-1}$ (the latter value comes from nonadiabatic calculations).

${ }^{b}$ Ref. [18]; ${ }^{c}$ Ref. [20]; ${ }^{d}$ Ref. [21].

TABLE V. Contributions to the dissociation energy $D_{0}$ and two selected most accurate experimental transitions in $\mathrm{D}_{2}\left(\right.$ in $\left.\mathrm{cm}^{-1}\right)$. There are additional $5 \cdot 10^{-4}$ relative uncertainties on $E^{(2)}, E^{(3)}$, and $E^{(4)}$ terms due to the $\mathrm{BO}$ approximation, which are included in the final result only.

\begin{tabular}{lccc}
\hline \hline Contrib. & $D_{0}$ & $S_{2}(2)$ & $Q_{1}(0)$ \\
\hline$E$ & $36749.09098(8)$ & $6241.12096(30)$ & $2993.61488(15)$ \\
$E^{(2)}$ & $-0.529170(1)$ & 0.040057 & 0.017677 \\
$E^{(3)}$ & $-0.1982(2)$ & $-0.03315(3)$ & $-0.01539(2)$ \\
$E^{(4)}$ & $-0.002096(6)$ & -0.000299 & -0.000139 \\
$E^{(5)}$ & $0.00012(6)$ & $0.000019(10)$ & $0.000009(5)$ \\
$E_{\mathrm{FS}}$ & -0.000204 & -0.000032 & -0.000015 \\
Total & $36748.3614(4)$ & $6241.12755(30)$ & $2993.61702(15)$ \\
Exp. & $36748.36286(68)^{a}$ & $6241.12764(2)^{b}$ & $2993.61706(15)^{c}$ \\
Diff. & 0.00146 & 0.00009 & 0.00004 \\
\hline \hline
\end{tabular}

${ }^{a}$ Ref. [22]; ${ }^{b}$ Ref. [23]; ${ }^{c}$ Ref. [21]. 